AEI-2005-137

\title{
One loop photon-graviton mixing in an electromagnetic field: Part 2
}

\author{
F. Bastianelli ${ }^{a, b}$, U. Nucamendi ${ }^{c}$, C. Schubert ${ }^{a, c}$, V. M. Villanueva ${ }^{a, c}$ \\ a Max-Planck-Institut für Gravitationsphysik, Albert-Einstein-Institut, \\ Mühlenberg 1, D-14476 Potsdam, Germany \\ ${ }^{b}$ Dipartimento di Fisica, Università di Bologna and INFN, Sezione di \\ Bologna, Via Irnerio 46, I-40126 Bologna, Italy \\ c Instituto de Fúsica y Matemáticas \\ Universidad Michoacana de San Nicolás de Hidalgo \\ Edificio C-3, Apdo. Postal 2-82 \\ C.P. 58040, Morelia, Michoacán, México
}

\begin{abstract}
In part 1 of this series compact integral representations had been obtained for the one-loop photon-graviton amplitude involving a charged spin 0 or spin $1 / 2$ particle in the loop and an arbitrary constant electromagnetic field. In this sequel, we study the structure and magnitude of the various polarization components of this amplitude on-shell. Explicit expressions are obtained for a number of limiting cases.
\end{abstract}




\section{Introduction}

As has been recognized many years ago 1, 2, the quantized Einstein-Maxwell theory predicts the process of photon-graviton conversion in an electromagnetic field. The tree level vertex for this amplitude is (see appendix A)

$$
\frac{1}{2} \kappa h_{\mu \nu}\left(F^{\mu \alpha} f_{\alpha}^{\nu}+f_{\alpha}^{\mu} F^{\nu \alpha}\right)-\frac{1}{4} \kappa h_{\mu}^{\mu} F^{\alpha \beta} f_{\alpha \beta} .
$$

Here $h_{\mu \nu}$ denotes the graviton, $f_{\mu \nu}$ the photon, and $F^{\mu \nu}$ the external field. $\kappa$ is the gravitational coupling constant. The corresponding photon-graviton vertex in momentum space reads $-\frac{i}{2} \kappa C^{\mu \nu, \alpha}$, where

$$
C^{\mu \nu, \alpha}=(F \cdot k)^{\alpha} \eta^{\mu \nu}+F^{\mu \alpha} k^{\nu}+F^{\nu \alpha} k^{\mu}-(F \cdot k)^{\mu} \eta^{\nu \alpha}-(F \cdot k)^{\nu} \eta^{\mu \alpha} .
$$

This interaction leads, assuming sufficient coherence of propagation, to photongraviton oscillations which are analogous to the better-known neutrino flavour [3] and photon-axion oscillations [4, 5, 6]. The true eigenstates of propagation in a background field will in general be certain mixtures of photon and graviton states. Determining these eigenstates and their dispersion relations requires, at tree level and in Fourier space, the diagonalization of the following matrix (see eqs. A.6, A.7), and A.8):

$$
\left(\begin{array}{cc}
\eta^{\alpha \beta} k^{2}-k^{\alpha} k^{\beta} & \frac{i}{2} \kappa C^{\kappa \lambda, \alpha} \\
\frac{i}{2} \kappa C^{\mu \nu, \beta} & \frac{k^{2}}{4}\left(\eta^{\mu \kappa} \eta^{\nu \lambda}+\eta^{\mu \lambda} \eta^{\nu \kappa}-2 \eta^{\mu \nu} \eta^{\kappa \lambda}+\ldots\right)
\end{array}\right)\left(\begin{array}{c}
a_{\beta}(k) \\
h_{\kappa \lambda}(k)
\end{array}\right)=0
$$

Here $a_{\beta}(k)$ represents the photon and $h_{\kappa \lambda}(k)$ the graviton. For many cases of physical interest, this problem can be simplified assuming the field to be homogeneous or near-homogeneous, and the modified dispersion relations to be close to the vacuum ones. An efficient formalism for calculating the evolution of the photon-graviton or photon-axion system under these conditions was developed in 6 .

Due to the smallness of the gravitational coupling $\kappa$, the photon-graviton mixing case has received less attention than the photon-axion one. Nevertheless, a number of authors have studied possible observable effects [2, 6, 7, 8, 9, 10, 11. The chances of observing this process in the laboratory appear very remote. As with other processes involving very small couplings, 
the natural setting is astrophysics where one can hope to at least partially compensate this smallness by large field strengths or exposure times. In principle, any process based on photon-axion conversion in a field (see, e.g., [12, 13, 14, 15, 16, 17, 18]) must have an analogue based on photon-graviton conversion. In [6] photon-graviton conversion was considered in a pulsar field, as well as in the galactic magnetic field, but the effect was found to be very small. Photon-graviton conversion in a primordial magnetic field has been proposed as a possible contribution to the cosmic microwave background anisotropy [8, 9]. However, taking plasma effects into account renders the effect negligible [10].

A natural enhancement of the photon-graviton oscillation occurs in theories with extra dimensions [19] due to the existence of an infinite tower of Kaluza-Klein gravitons. In [20] both the effect of the photon-graviton oscillation on the cosmic microwave background and the conversion in a pulsar background were reconsidered in this context, but the enhancement was found to be insufficient to lift these effects into the observable range.

To our knowledge, the photon-graviton process has previously been studied only at the tree level. In the first part of this series [21] (referred to as 'part 1' in the following) we considered the one-loop corrections to this amplitude due to massive charged spin 0 (denoted $\bar{\Pi}_{\text {scal }}^{\mu \nu, \alpha}$ ) and spin $1 / 2$ particles (denoted $\left.\bar{\Pi}_{\text {spin }}^{\mu \nu, \alpha}\right)$ in the loop (fig. 1).

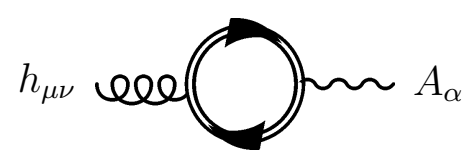

Figure 1: One-loop photon-graviton amplitude in a constant field. The double line represents the propagator of a charged scalar or spin $\frac{1}{2}$ particle in a constant field.

Our motivation for considering this loop correction is twofold. First, the dispersion relation (1.3) will be modified in the following way by the 
one-loop contributions (see eqs. (A.6), (A.7), and (A.8)):

$$
\begin{aligned}
& \left(\begin{array}{cc}
\eta^{\alpha \beta} k^{2}-k^{\alpha} k^{\beta}-\bar{\Pi}^{\alpha, \beta} & \frac{i}{2} \kappa C^{\kappa \lambda, \alpha}-\bar{\Pi}^{\kappa \lambda, \alpha} \\
\frac{i}{2} \kappa C^{\mu \nu, \beta}-\bar{\Pi}^{\mu \nu, \beta} & \frac{k^{2}}{4}\left(\eta^{\mu \kappa} \eta^{\nu \lambda}+\eta^{\mu \lambda} \eta^{\nu \kappa}-2 \eta^{\mu \nu} \eta^{\kappa \lambda}+\ldots\right)-\bar{\Pi}^{\mu \nu, \kappa \lambda}
\end{array}\right)\left(\begin{array}{c}
a_{\beta}(k) \\
h_{\kappa \lambda}(k)
\end{array}\right) \\
& \quad=0 .
\end{aligned}
$$

This equation involves the full one-loop photon-photon, photon-graviton and graviton-graviton amplitudes, computed in the constant external field, summed over all possible loop particles (including, e.g., photons and gluons for the graviton propagator). As is well-known, already in the pure QED case the one-loop corrected dispersion relation in an external field

$$
\left[\eta^{\mu \nu} k^{2}-k^{\mu} k^{\nu}-\Pi^{\mu, \nu}(k)\right] a_{\nu}(k)=0
$$

leads to highly nontrivial deviations from the vacuum case [22, 223, 24, 25, 26, 27, 28, 29, 30, 31, 32, 33. We believe that it will be very instructive to generalize this study to the mixed photon-graviton system (1.4), particularly considering the fact that in curved backgrounds superluminal phase velocities are known to occur [34, 35, 36]. See also [37] for recent studies on the photon vacuum polarization in curved space at arbitrary frequencies and related investigations on a possible breakdown of microcausality.

Second, while the tree level interaction term (1.1) depends linearly on the background field, the one-loop corrections depend nontrivially on the field strength as well as on the photon/graviton energy. Thus, although at linear order in $F_{\mu \nu}$ the one-loop corrections are down by an explicit factor of $\alpha$ compared to the tree level term, it is a priori conceivable that for sufficiently large fields and some range of photon energies the one-loop amplitudes would dominate over the tree level one.

The plan of this paper is as follows. In section 2 we find choices of physical polarizations well-adapted to the structure of the worldline parameter integrals obtained in part 1 . In section 3 we specialize to the on-shell case, and introduce some convenient notation. In section 4 we consider the purely magnetic case, and explicitly evaluate the amplitude for three ranges of parameters: in 4.1 we present a direct numerical evaluation for arbitrary field strength and photon/graviton energies below threshold; in 4.2 closed-form results are found for the zero energy limits; in 4.3 we consider the case of a weak field but arbitrary energies.

It seems that photon-graviton conversion so far has been studied only for the magnetic field case. Although the physical relevance of this process 
is even more hypothetical in the electric field case, in part 1 we kept the electric field component since, quite generally, in the worldline formalism calculations in a general electromagnetic field are not substantially more difficult than in a purely magnetic field [38, 39]. In this sequel, too, we shortly consider the electric field case in section 5, and present results for two cases where the electric result can easily be inferred from the magnetic one, namely the zero energy and weak field cases. We summarize our results in section 6 ,

\section{Polarization decomposition of the amplitude}

Let us now project the photon-graviton amplitude on physical polarizations. We need to choose two photon polarization vectors $\varepsilon_{1,2}$ such that

$$
\varepsilon_{i}^{\mu} \varepsilon_{j \mu}=\delta_{i j}, \quad k_{\mu} \varepsilon_{i}^{\mu}=0 .
$$

Similarly, we need two symmetric, traceless, and transverse graviton polarization tensors $\varepsilon_{1,2}^{\mu \nu}$ :

$$
\varepsilon_{i}^{\mu \nu} \varepsilon_{j \mu \nu}=2 \delta_{i j}, \quad \varepsilon_{i}^{\mu \nu}=\varepsilon_{i}^{\nu \mu}, \quad \varepsilon_{i \mu}^{\mu}=0, \quad k_{\mu} \varepsilon_{i}^{\mu \nu}=0 .
$$

As we will see, substantial simplifications can be achieved by choosing polarization vectors which are adapted to the background field. In part 1 we had, following [38, 39], written the worldline Green functions using the matrix basis $\hat{\mathcal{Z}}_{ \pm}, \hat{\mathcal{Z}}_{ \pm}^{2}$

$$
\begin{aligned}
\hat{\mathcal{Z}}_{+}^{\mu \nu} & =\frac{a F^{\mu \nu}-b \tilde{F}^{\mu \nu}}{a^{2}+b^{2}}, \quad \hat{\mathcal{Z}}_{-}^{\mu \nu}=-i \frac{b F^{\mu \nu}+a \tilde{F}^{\mu \nu}}{a^{2}+b^{2}} \\
\left(\hat{\mathcal{Z}}_{+}^{2}\right)^{\mu \nu} & =\frac{F^{\mu \lambda} F_{\lambda}^{\nu}-b^{2} \eta^{\mu \nu}}{a^{2}+b^{2}}, \quad\left(\hat{\mathcal{Z}}_{-}^{2}\right)^{\mu \nu}=-\frac{F^{\mu \lambda} F_{\lambda}^{\nu}+a^{2} \eta^{\mu \nu}}{a^{2}+b^{2}} .
\end{aligned}
$$

Here $\tilde{F}_{\mu \nu}=\frac{1}{2} \varepsilon_{\mu \nu \alpha \beta} F^{\alpha \beta}$ is the dual field strength tensor 1 and $a, b$ are related to the two standard Maxwell invariants by $a^{2}-b^{2}=B^{2}-E^{2}, a b=\mathbf{E} \cdot \mathbf{B}$.

\footnotetext{
${ }^{1}$ We work in Minkowski space with $\eta^{\mu \nu}=\operatorname{diag}(-+++)$ and $\varepsilon^{0123}=1$.
} 
Note the following properties of this basis [38, 39],

$$
\begin{aligned}
\hat{\mathcal{Z}}_{ \pm}^{3} & =-\hat{\mathcal{Z}}_{ \pm}, \\
\hat{\mathcal{Z}}_{+} \cdot \hat{\mathcal{Z}}_{-} & =0 .
\end{aligned}
$$

We now use this basis to define polarization vectors as follows ${ }^{2}$,

$$
\varepsilon_{ \pm}^{\mu} \equiv \frac{\left(\hat{\mathcal{Z}}_{ \pm} \cdot k\right)^{\mu}}{\lambda_{ \pm}}
$$

Here the $\lambda_{ \pm}$'s are normalization factors,

$$
\begin{aligned}
& \lambda_{+} \equiv \sqrt{\left(\hat{\mathcal{Z}}_{+} \cdot k\right) \cdot\left(\hat{\mathcal{Z}}_{+} \cdot k\right)}=\sqrt{-k \cdot\left(\hat{\mathcal{Z}}_{+}^{2}\right) \cdot k}, \\
& \lambda_{-} \equiv-i \sqrt{-\left(\hat{\mathcal{Z}}_{-} \cdot k\right) \cdot\left(\hat{\mathcal{Z}}_{-} \cdot k\right)}=-i \sqrt{k \cdot\left(\hat{\mathcal{Z}}_{-}^{2}\right) \cdot k} .
\end{aligned}
$$

Together with the orthogonality relation (2.5) they ensure that $\varepsilon_{+}, \varepsilon_{-}$satisfy the conditions (2.1). Both $\lambda_{ \pm}$can vanish; in this case the corresponding polarization vector is lightlike and cannot be normalized. We also note that

$$
\lambda_{+}^{2}+\lambda_{-}^{2}=k^{2}
$$

(from (2.3)). The explicit form of $\varepsilon_{ \pm}$in terms of $\mathbf{E}$ and $\mathbf{B}$ is rather complicated in the general case. However, as usual things simplify considerably if one specializes to a Lorentz system where $\mathbf{E}$ and $\mathbf{B}$ are both pointing along the positive $\mathrm{z}$ - axis, $\mathbf{E}=(0,0, E), \mathbf{B}=(0,0, B)$. This implies that $a=B$ and $b=E$. Here the dependence of $\lambda_{ \pm}, \varepsilon_{ \pm}$on the field magnitudes drops out, leaving only a memory of the field direction:

$$
\lambda_{+}=\sqrt{\left(k^{1}\right)^{2}+\left(k^{2}\right)^{2}} \equiv \lambda_{\perp}, \quad \lambda_{-}=-i \sqrt{\left(k^{0}\right)^{2}-\left(k^{3}\right)^{2}} \equiv \lambda_{\|}
$$

and

\footnotetext{
${ }^{2}$ For the photon polarizations this basis has been introduced in 32 with different conventions and notations (in particular, our $b$ corresponds to $-b$ there).
} 


$$
\varepsilon_{+}^{\mu}=\frac{\left(0, k^{2},-k^{1}, 0\right)}{\sqrt{\left(k^{1}\right)^{2}+\left(k^{2}\right)^{2}}} \equiv \varepsilon_{\perp}^{\mu}, \quad \varepsilon_{-}^{\mu}=\frac{\left(k^{3}, 0,0, k^{0}\right)}{\sqrt{\left(k^{0}\right)^{2}-\left(k^{3}\right)^{2}}} \equiv \varepsilon_{\|}^{\mu} .
$$

The subscripts $\perp, \|$ refer to the field direction. Such a Lorentz system exists provided that $\mathbf{E} \cdot \mathbf{B}>0$. The case $\mathbf{E} \cdot \mathbf{B}<0$ differs from this only by a parity transformation, but the case $\mathbf{E} \cdot \mathbf{B}=0$ needs to be considered separately: This case can for $E>B$ be transformed into the purely electric and for $B>E$ into the purely magnetic field case. In both cases $\lambda_{ \pm}$and $\varepsilon_{ \pm}$are the same as in (2.9), 2.10) if the $\mathrm{z}$-axis is chosen as the field direction. The remaining possibility is that $\mathbf{E} \cdot \mathbf{B}=0$ and $E=B$, the "crossed field" case; here $a=b=0$ and the above basis cannot be used.

To simplify further, without loss of generality we shall assume that the photon propagation is in the $y-z$ plane $\left(k^{1}=0, k^{2}>0\right)$.

It will be useful to construct also the graviton polarizations using the same building blocks. We define

$$
\begin{aligned}
\varepsilon^{\oplus \mu \nu} & \equiv \varepsilon^{+\mu} \varepsilon^{+\nu}-\varepsilon^{-\mu} \varepsilon^{-\nu} \\
\varepsilon^{\otimes \mu \nu} & \equiv \varepsilon^{+\mu} \varepsilon^{-\nu}+\varepsilon^{-\mu} \varepsilon^{+\nu}
\end{aligned}
$$

Then the conditions (2.2) are fulfilled as a consequence of (2.1).

This basis is extremely convenient since, when contracting $\Pi_{\text {scal,spin }}^{\mu \nu, \alpha}$ with these polarization vectors/tensors, many terms in the integral representations obtained in part 1 (eqs. (3.13) and (4.11) there) drop out on account of the orthogonality relation 2.5 and the antisymmetry of $\hat{\mathcal{Z}}_{ \pm}$. In particular, all terms involving a factor of $\dot{\mathcal{S}}_{B 12} \cdot k$ or $\ddot{\mathcal{S}}_{B 12} \cdot k$ will vanish.

We remark that a more standard, but less convenient, basis would be obtained by removing the time component of $\varepsilon_{\|}$by a longitudinal shift,

$$
\tilde{\varepsilon}_{\|}^{\mu}=\varepsilon_{\|}^{\mu}-\frac{k^{3} k^{\mu}}{k^{0} \sqrt{\left(k^{0}\right)^{2}-\left(k^{3}\right)^{2}}}
$$

The equivalence of these two choices may not seem obvious for the gravitational part, since neither $C^{\mu \nu, \alpha}$ nor $\bar{\Pi}_{\text {scal,spin }}^{\mu \nu, \alpha}$ are transversal in the graviton indices. However, using the gravitational Ward identity ((A.13) of part 1) it is easily shown that the shift makes no difference for the matrix elements. 
In the following, we will denote

$$
C^{A a} \equiv \varepsilon_{\mu \nu}^{A} C^{\mu \nu, \alpha} \varepsilon_{\alpha}^{a}
$$

etc. where $A=\oplus, \otimes$ and $a=\perp, \|$. At the tree level, one obtains the following simple result:

$$
\begin{aligned}
C^{\oplus \perp} & =-2 B \lambda_{\perp}, \\
C^{\oplus \|} & =2 i E \lambda_{\|}, \\
C^{\otimes \perp} & =-2 i E \lambda_{\|}, \\
C^{\otimes \|} & =-2 B \lambda_{\perp} .
\end{aligned}
$$

The fact that for a purely magnetic (electric) field $\varepsilon^{\oplus}$ couples only to $\varepsilon^{\perp}$ $\left(\varepsilon^{\|}\right)$and $\varepsilon^{\otimes}$ only to $\varepsilon^{\|}\left(\varepsilon^{\perp}\right)$ is a consequence of CP invariance (see [6]). It must therefore also hold for the loop corrections.

For the one-loop correction, using the polarization choices (2.6), 2.11) in (3.13) resp. (4.11) of part 1 yields the following:

$$
\begin{array}{r}
\bar{\Pi}_{\text {scal }}^{A a}=\frac{e \kappa}{64 \pi^{2}} \int_{0}^{\infty} \frac{d s}{s} \mathrm{e}^{-i s m^{2}}\left\{\frac{z_{+} z_{-}}{\sinh \left(z_{+}\right) \sinh \left(z_{-}\right)}\right. \\
\left.\times \int_{0}^{1} d u \mathrm{e}^{-i s \Phi} \tilde{J}_{\text {scal }}^{A a}+\frac{2}{3} i e C^{A a}\right\}, \\
\bar{\Pi}_{\text {spin }}^{A a}=-\frac{e \kappa}{32 \pi^{2}} \int_{0}^{\infty} \frac{d s}{s} \mathrm{e}^{-i s m^{2}}\left\{\frac{z_{+} z_{-}}{\tanh \left(\mathrm{z}_{+}\right) \tanh \left(\mathrm{z}_{-}\right)}\right. \\
\left.\times \int_{0}^{1} d u \mathrm{e}^{-i s \Phi} \tilde{J}_{\text {spin }}^{A a}-\frac{4}{3} i e C^{A a}\right\},
\end{array}
$$

where now $z_{+}=i e B s, z_{-}=-e E s$ and (repeated indices $a, b, \ldots$ are to be summed over \pm )

$$
\Phi=-\frac{1}{2} \frac{\bar{A}^{a}}{z^{a}} \lambda_{a}^{2}
$$




$$
\begin{aligned}
\tilde{J}_{\text {scal }}^{\oplus \perp}= & \frac{2}{s}\left(z_{+} A_{B 11}^{+}-z_{-} A_{B 11}^{-}\right) \bar{A}_{B 12}^{+} \lambda_{+} \\
& +\frac{2}{s} z_{+}\left[\left(S_{B 12}^{+}\right)^{2}-\bar{A}_{B 12}^{+}\left(A_{B 12}^{+}+\frac{1}{z_{+}}\right)\right] \lambda_{+} \\
& +i \bar{A}_{B 12}^{+} \lambda_{+}\left[\left(\left(S_{B 12}^{+}\right)^{2}-\left(\bar{A}_{B 12}^{+}\right)^{2}\right) \lambda_{+}^{2}+\left(S_{B 12}^{+} S_{B 12}^{-}+\left(\bar{A}_{B 12}^{-}\right)^{2}\right) \lambda_{-}^{2}\right], \\
\tilde{J}_{\text {scal }}^{\otimes \perp}= & \frac{2}{s}\left[S_{B 12}^{+} z_{-} S_{B 12}^{-}-\bar{A}_{B 12}^{-} z_{+}\left(A_{B 12}^{+}+\frac{1}{z_{+}}\right)\right] \lambda_{-} \\
& +i \bar{A}_{B 12}^{-} \lambda_{-}\left[S_{B 12}^{+} S_{B 12}^{a} \lambda_{a}^{2}-2\left(\bar{A}_{B 12}^{+}\right)^{2} \lambda_{+}^{2}\right],
\end{aligned}
$$

$$
\begin{aligned}
\tilde{J}_{\text {spin }}^{\oplus \perp}= & \frac{2}{s}\left[z_{+}\left(A_{B 11}^{+}-A_{F 11}^{+}\right)-z_{-}\left(A_{B 11}^{-}-A_{F 11}^{-}\right)\right]\left(\bar{A}_{B 12}^{+}+A_{F 22}^{+}\right) \lambda_{+} \\
& +\frac{2}{s} z_{+}\left[\left(S_{B 12}^{+}\right)^{2}-\left(S_{F 12}^{+}\right)^{2}+\left(A_{F 12}^{+}\right)^{2}-\left(\bar{A}_{B 12}^{+}+A_{F 11}^{+}\right)\left(A_{B 12}^{+}+\frac{1}{z_{+}}\right)\right] \lambda_{+} \\
& +i \bar{A}_{B 12}^{+} \lambda_{+}\left(S_{B 12}^{+} S_{B 12}^{a}-S_{F 12}^{+} S_{F 12}^{a}\right) \lambda_{a}^{2} \\
& -i \bar{A}_{B 12}^{+}\left[\left(\bar{A}_{B 12}^{+}+A_{F 11}^{+}\right)^{2}-\left(A_{F 12}^{+}\right)^{2}\right] \lambda_{+}^{3} \\
& +i \bar{A}_{B 12}^{-}\left[\left(\bar{A}_{B 12}^{-}+A_{F 11}^{-}\right)\left(\bar{A}_{B 12}^{+}+A_{F 11}^{+}\right)-A_{F 12}^{-} A_{F 12}^{+}\right] \lambda_{+} \lambda_{-}^{2}, \\
\tilde{J}_{\mathrm{spin}}^{\otimes \perp}= & \frac{2}{s}\left[\left(S_{B 12}^{+} S_{B 12}^{-}-S_{F 12}^{+} S_{F 12}^{-}\right) z_{-}-\left(\bar{A}_{B 12}^{-}+A_{F 11}^{-}\right) z_{+}\left(A_{B 12}^{+}+\frac{1}{z_{+}}\right)+z_{+} A_{F 12}^{+} A_{F 12}^{-}\right] \lambda_{-} \\
& +i \bar{A}_{B 12}^{-} \lambda_{-}\left(S_{B 12}^{+} S_{B 12}^{a}-S_{F 12}^{+} S_{F 12}^{a}\right) \lambda_{a}^{2} \\
& -i \bar{A}_{B 12}^{-}\left[\left(\bar{A}_{B 12}^{+}+A_{F 11}^{+}\right)^{2}-\left(A_{F 12}^{+}\right)^{2}\right] \lambda_{+}^{2} \lambda_{-} \\
& -i \bar{A}_{B 12}^{+}\left[\left(\bar{A}_{B 12}^{-}+A_{F 11}^{-}\right)\left(\bar{A}_{B 12}^{+}+A_{F 11}^{+}\right)-A_{F 12}^{-} A_{F 12}^{+}\right] \lambda_{+}^{2} \lambda_{-} \cdot
\end{aligned}
$$

The remaining components are obtained using the symmetry

$$
\begin{aligned}
& \tilde{J}_{\text {scal,spin }}^{\oplus \|}=-\tilde{J}_{\text {scal,spin }}^{\oplus \perp}(+\leftrightarrow-), \\
& \tilde{J}_{\text {scal,spin }}^{\otimes \|}=\tilde{J}_{\text {scal,spin }}^{\otimes \perp}(+\leftrightarrow-) .
\end{aligned}
$$


The integrands are written in terms of the standard worldline functions (see (3.21) and (4.9) of part 1)

$$
\begin{aligned}
S_{B 12}^{ \pm} & =\frac{\sinh \left(z_{ \pm}(1-2 u)\right)}{\sinh \left(z_{ \pm}\right)} \\
A_{B 12}^{ \pm} & =\frac{\cosh \left(z_{ \pm}(1-2 u)\right)}{\sinh \left(z_{ \pm}\right)}-\frac{1}{z_{ \pm}} \\
A_{B 11}^{ \pm}=A_{B 22}^{ \pm} & =\operatorname{coth}\left(\mathrm{z}_{ \pm}\right)-\frac{1}{\mathrm{z}_{ \pm}} \\
\bar{A}_{B 12}^{ \pm} & =A_{B 12}^{ \pm}-A_{B 11}^{ \pm}=\frac{\cosh \left(z_{ \pm}(1-2 u)\right)-\cosh \left(z_{ \pm}\right)}{\sinh \left(z_{ \pm}\right)} \\
S_{F 12}^{ \pm} & =\frac{\cosh \left(z_{ \pm}(1-2 u)\right)}{\cosh \left(z_{ \pm}\right)} \\
A_{F 12}^{ \pm} & =\frac{\sinh \left(z_{ \pm}(1-2 u)\right)}{\cosh \left(z_{ \pm}\right)} \\
A_{F 11}^{ \pm}= & \tanh \left(z_{ \pm}\right)
\end{aligned}
$$

Note that, as usual in this formalism [38, 39], the scalar loop integrands (2.17) are obtained from the spinor loop ones (2.18) simply by nullifying all fermionic worldline correlators $S_{F i j}^{ \pm}, A_{F i j}^{ \pm}$.

\section{On-shell amplitudes}

In vacuum at this point we would use the dispersion relation

$$
k^{2}=0
$$

The modifications of this relation due to gravitational corrections are not relevant for our present purposes, since they would produce terms of higher order in $\kappa$. However, this is less clear for the field-induced corrections to the electromagnetic $\Pi^{\mu, \nu}$. The question of under which conditions $(1.5)$ can still be well-approximated by (3.1) was, for the magnetic case, studied in [32. There it was shown that this is the case at least for moderate fields and frequencies, $B \leq O\left(B_{\mathrm{cr}}\right)$ and $\omega \leq O(m)$, where $B_{c r}=\frac{m^{2}}{e}$ denotes the "critical" magnetic field strength $\left(B_{c r}=4.4 \times 10^{9} \mathrm{~T}\right.$ for electrons). Here 
the restriction on the photon frequency is not very significant, since for frequencies beyond the pair creation threshold $\omega=2 m$ processes involving electron-positron pair creation become possible, and then are usually physically more relevant than the dispersive processes which we are concerned with here. To the contrary, the bound on the field strength may pose a restriction for applications to magnetars which are believed to carry field strengths up to several orders of magnitude higher than $B_{c r}$ [40].

In the following, we will assume that the use of $k^{2}=0$ is justified, and work out the consequences. Using $k^{0}=\omega=|\vec{k}|$ eqns. 2.9, 2.10 simplify to $\left(\right.$ with $\left.k^{1}=0\right)$

$$
\begin{aligned}
\varepsilon_{\perp}^{\mu} & =(0,1,0,0), \\
\varepsilon_{\|}^{\mu} & =\frac{1}{\sin \theta}(\cos \theta, 0,0,1),
\end{aligned}
$$

and

$$
\begin{aligned}
\lambda_{\perp} & =\omega \sin \theta, \\
\lambda_{\|} & =-i \omega \sin \theta,
\end{aligned}
$$

where $\theta$ is the angle between the $z$-axis (the field direction) and the direction of the photon propagation. Since in this approximation the amplitude depends on $\omega$ and $\theta$ only in the combination $\omega \sin \theta$, there is no point in keeping the dependence on $\theta$. We will therefore restrict ourselves in the following to the case $\sin \theta=1$ (propagation perpendicular to the field direction). For a general field we are then left with the four parameters $m, \omega, B$, and $E$. It will be convenient to work with the three dimensionless variables

$$
\begin{aligned}
\hat{\omega} & \equiv \frac{\omega}{m}, \\
\hat{B} & \equiv \frac{e B}{m^{2}}=\frac{B}{B_{c r}}, \\
\hat{E} & \equiv \frac{e E}{m^{2}}=\frac{E}{E_{c r}} .
\end{aligned}
$$


Here $E_{c r}=\frac{m^{2}}{e}$ denotes the electric "critical" field strength $\left(1.3 \times 10^{18} \mathrm{~V} / \mathrm{m}\right.$ for electrons). Similarly, for the calculation of the integrals it will be useful to change to the dimensionless proper-time variable $\hat{s} \equiv m^{2} s$. Moreover, as usual in this type of calculations we will change from $u$ to $v \equiv 1-2 u$.

Finally, since we wish to compare the one-loop and the tree level contributions, we normalize the former by the latter. Thus we will have to compute

$$
\begin{aligned}
\hat{\Pi}_{\text {scal }, \text { spin }}^{A a}(\hat{\omega}, \hat{B}, \hat{E}) & \equiv \operatorname{Re}\left(\frac{\bar{\Pi}_{\text {scal,spin }}^{A a}}{-\frac{i}{2} \kappa C^{A a}}\right) \\
& =\alpha \operatorname{Re} \int_{0}^{\infty} \frac{d \hat{s}}{\hat{s}} \mathrm{e}^{-i \hat{s}} \int_{0}^{1} d v \hat{\pi}_{\text {scal }, \text { spin }}^{A a}(\hat{s}, v, \hat{\omega}, \hat{B}, \hat{E})
\end{aligned}
$$

with dimensionless integrands $\hat{\pi}_{\mathrm{scal}, \mathrm{spin}}^{A a}$.

\section{The magnetic field case}

We specialize to the purely magnetic case, $E=0$. Then $z_{-}=0$ so that

$$
\begin{aligned}
S_{B 12}^{-} & =v, \\
S_{F 12}^{-} & =1, \\
A_{B 12}^{-} & =0, \\
A_{F 12}^{-} & =0, \\
\frac{\bar{A}_{B 12}^{-}}{z_{-}} & =\frac{1}{2}\left(v^{2}-1\right) .
\end{aligned}
$$

Using these identities in eqs. 2.17), 2.18, 2.19 one can immediately show that

$$
\Pi_{\mathrm{scal}, \mathrm{spin}}^{\oplus \|}=\Pi_{\mathrm{scal}, \mathrm{spin}}^{\otimes \perp}=0
$$

This is in accordance with the CP analysis mentioned above. 
The integrands of the nonvanishing components are, using (3.2), 3.3,

$$
\begin{aligned}
\hat{\pi}_{\mathrm{scal}}^{\oplus \perp}=\frac{1}{8 \pi} & \left\{\frac{z_{+}}{\sinh \left(z_{+}\right)} \exp \left[z_{+}\left(\frac{\bar{A}_{B 12}^{+}}{z_{+}}+\frac{1}{2}\left(1-v^{2}\right)\right) \frac{\hat{\omega}^{2}}{2 \hat{B}}\right]\right. \\
& \left.\times\left[\left(S_{B 12}^{+}\right)^{2}-\bar{A}_{B 12}^{+}\left(\bar{A}_{B 12}^{+}+\frac{1}{z_{+}}\right)+\bar{A}_{B 12}^{+}\left(\left(S_{B 12}^{+}\right)^{2}-\left(\bar{A}_{B 12}^{+}\right)^{2}-v S_{B 12}^{+}\right) \frac{\hat{\omega}^{2}}{2 \hat{B}}\right]-\frac{2}{3}\right\}, \\
\hat{\pi}_{\mathrm{scal}}^{\otimes \|}=\frac{1}{8 \pi} & \left\{\frac{z_{+}}{\sinh \left(z_{+}\right)} \exp \left[z_{+}\left(\frac{\bar{A}_{B 12}^{+}}{z_{+}}+\frac{1}{2}\left(1-v^{2}\right)\right) \frac{\hat{\omega}^{2}}{2 \hat{B}}\right]\right. \\
& \left.\times\left[v S_{B 12}^{+}-\frac{\bar{A}_{B 12}^{+}}{z_{+}}+\bar{A}_{B 12}^{+} v\left(S_{B 12}^{+}-v\right) \frac{\hat{\omega}^{2}}{2 \hat{B}}\right]-\frac{2}{3}\right\}
\end{aligned}
$$

for the scalar case and

$$
\begin{aligned}
\hat{\pi}_{\mathrm{spin}}^{\oplus \perp}=- & \frac{1}{4 \pi}\left\{\frac{z_{+}}{\tanh \left(\mathrm{z}_{+}\right)} \exp \left[z_{+}\left(\frac{\bar{A}_{B 12}^{+}}{z_{+}}+\frac{1}{2}\left(1-v^{2}\right)\right) \frac{\hat{\omega}^{2}}{2 \hat{B}}\right]\right. \\
\times & {\left[\left(S_{B 12}^{+}\right)^{2}-\left(S_{F 12}^{+}\right)^{2}+\left(A_{F 12}^{+}\right)^{2}-\left(\bar{A}_{B 12}^{+}+A_{F 11}^{+}\right)\left(\bar{A}_{B 12}^{+}+\frac{1}{z_{+}}+A_{F 11}^{+}\right)\right.} \\
& \left.+\bar{A}_{B 12}^{+}\left(\left(S_{B 12}^{+}\right)^{2}-\left(S_{F 12}^{+}\right)^{2}-\left(\bar{A}_{B 12}^{+}+A_{F 11}^{+}\right)^{2}+\left(A_{F 12}^{+}\right)^{2}-v S_{B 12}^{+}+S_{F 12}^{+}\right) \frac{\hat{\omega}^{2}}{2 \hat{B}}\right] \\
& \left.\quad+\frac{4}{3}\right\}, \\
\hat{\pi}_{\text {spin }}^{\otimes \|}=- & \frac{1}{4 \pi}\left\{\frac{z_{+}}{\tanh \left(\mathrm{z}_{+}\right)} \exp \left[z_{+}\left(\frac{\bar{A}_{B 12}^{+}}{z_{+}}+\frac{1}{2}\left(1-v^{2}\right)\right) \frac{\hat{\omega}^{2}}{2 \hat{B}}\right]\right. \\
\times & {\left[v S_{B 12}^{+}-S_{F 12}^{+}-\frac{1}{z_{+}}\left(\bar{A}_{B 12}^{+}+A_{F 11}^{+}\right)+\bar{A}_{B 12}^{+}\left(v S_{B 12}^{+}-S_{F 12}^{+}+1-v^{2}\right) \frac{\hat{\omega}^{2}}{2 \hat{B}}\right] } \\
& \left.+\frac{4}{3}\right\}
\end{aligned}
$$

for the spinor case. Here $z_{+}=i \hat{B} \hat{s}$ and $S_{B 12}^{+}, \bar{A}_{B 12}^{+}$etc. are as in 2.20 with $1-2 u$ replaced by $v$.

The resulting integrals (3.5) have a structure similar to the parameter integrals for the photon vacuum polarization in a constant magnetic field obtained by Tsai and Erber [29]. Evaluating this type of integral for the whole range of field strengths and photon energies is known to be difficult [29, $31,41,42,33$, and in fact appears to have never been done in full generality 
for the photon-photon case. For energies below the pair creation energy a direct numerical calculation is unproblematic after rotating to Euclidean proper time, $\hat{T} \equiv i \hat{s}$. From eqs. 3.5$),(4.3),(4.4),(2.20)$ it is evident that the $v$ - integral is always finite, and the integrand of the resulting $\hat{T}$ - integral is exponentially falling for small $\hat{\omega}$. The pair creation threshold $\hat{\omega}_{\text {cr }}$ can be recognized precisely through the fact that for $\hat{\omega}>\hat{\omega}_{\text {cr }}$ the $\hat{T}-$ integral becomes divergent at large $\hat{T}$. Thus, analyzing the asymptotic behaviour of the integrands for large $\hat{T}$ at fixed $B$ one finds that, for the scalar case, the critical value $\hat{\omega}_{\text {cr }}$ is the same for both polarization components, while for the spinor case it depends on the polarization:

$$
\begin{aligned}
\hat{\omega}_{\mathrm{cr}, \mathrm{scal}}^{\oplus \perp}= & \hat{\omega}_{\mathrm{cr}, \mathrm{scal}}^{\otimes \|} \\
\hat{\omega}_{\mathrm{cr}, \mathrm{spin}}^{\oplus \perp} & =2 \sqrt{1+\hat{B}}, \\
\hat{\omega}_{\mathrm{cr}, \mathrm{spin}}^{\otimes \|} & =2 .
\end{aligned}
$$

This divergence signals the onset of real pair creation, although to study the pair creation process itself one would have to consider the full photongraviton polarization matrix (see (1.4) above). All four threshold values (4.5) agree with what one finds for the corresponding photon-photon amplitudes [22, 26, 38, 42].

Beyond the critical energy Euclidean proper time cannot be used any more, while a numerical integration in the original proper - time variable $\hat{s}$ poses enormous difficulties, due to the combined effect of the oscillatory behaviour of the universal exponential factor and the poles of the trigonometric prefactor functions.

Thus we will restrict our investigation to the two regimes which have also been well-studied in the photon-photon case, namely (i) photon energies below the pair creation threshold with arbitrary $B$ and (ii) arbitrary photon energies at low field strength. Special attention will be given to the limit of zero photon energy, since, as we will see, here one can obtain the amplitudes in closed form.

\subsection{Photon energies below threshold}

As mentioned above, below the pair creation threshold the parameter integrals 3.5 are, after a Wick rotation $\hat{T}=i \hat{s}$, suitable for a direct numerical evaluation at arbitrary magnetic field strength. Fig. 2 shows the results of 
such an evaluation, using MATHEMATICA, for the spinor loop amplitude ratio $\hat{\Pi}_{\text {spin }}^{\oplus \perp}(\hat{\omega}, \hat{B})$. The amplitude ratio is shown for field strengths in the range $B_{\mathrm{cr}}<B<10^{4} B_{\mathrm{cr}}$ and photon/graviton energies $\hat{\omega} \leq 2$. A global factor of $-\frac{\alpha}{4 \pi}$ has been omitted.

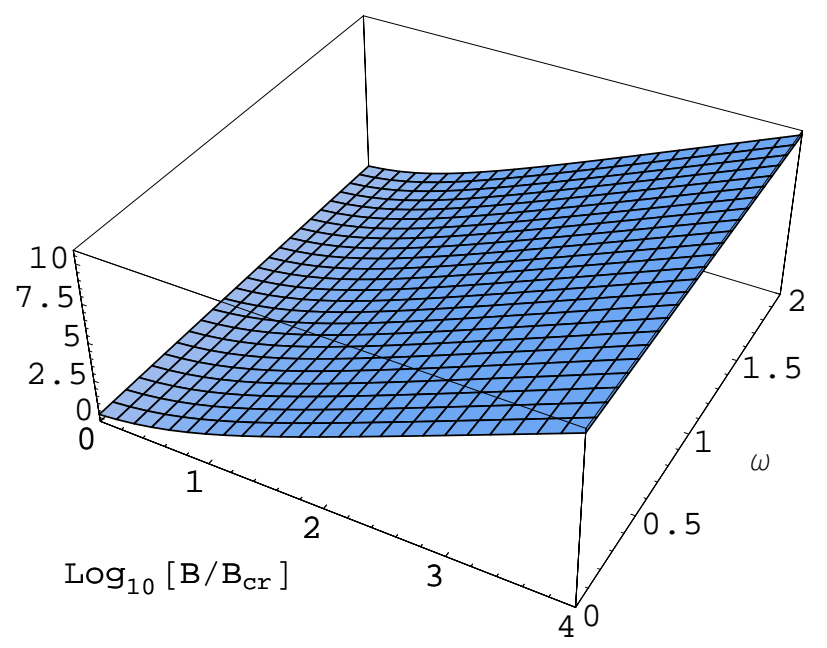

Figure 2: Numerical plot of the amplitude $\hat{\Pi}_{\text {spin }}^{\oplus \perp}(\hat{\omega}, \hat{B})$ for $\hat{B}$ between 1 and $10^{4}$ and $\hat{\omega} \leq 2$. A global factor of $-\frac{\alpha}{4 \pi}$ has been omitted.

The corresponding plots for $\hat{\Pi}_{\text {spin }}^{\otimes \|}$ and $\hat{\Pi}_{\text {scal }}^{\oplus \perp}, \hat{\Pi}_{\text {scal }}^{\otimes \|}$ are very similiar for photon/graviton energies below the corresponding thresholds (the numerical integration becomes unstable for $\omega$ too close to $\omega_{\text {crit }}$ ). In particular, all four amplitude ratios display a logarithmic growth in $B$ for large $B \gg B_{\mathrm{cr}}$. And indeed, for fixed $\omega<\omega_{\text {cr }}$ and $\hat{B} \rightarrow \infty$ it is easy to show the following asymptotic behaviour of (the nonvanishing components of) $\hat{\Pi}_{\text {scal,spin }}^{A a}(\hat{\omega}, \hat{B})$,

$$
\begin{array}{ll}
\hat{\Pi}_{\text {scal }}^{A a}(\hat{\omega}, \hat{B}) & \stackrel{\hat{B} \rightarrow \infty}{\sim}-\frac{\alpha}{12 \pi} \ln (\hat{B}), \\
\hat{\Pi}_{\text {spin }}^{A a}(\hat{\omega}, \hat{B}) & \stackrel{\hat{B} \rightarrow \infty}{\sim}-\frac{\alpha}{3 \pi} \ln (\hat{B}) .
\end{array}
$$

Thus this asymptotic behaviour is independent of the photon energy, as well as of the polarization choice. Moreover, it is easy to see that it relates directly to the renormalization terms in the integrands $(4.3),(4.4)$. Thus we recognize here a connection between the short-distance behaviour and the 
strong-field limit of this amplitude which is familiar from the photon-photon case, as well as other external field processes [43, 44].

\subsection{Zero photon energy at arbitrary magnetic field strength}

Closed-form results can be obtained in the zero energy limit. Setting $\hat{\omega}=0$

in (4.3), 4.4 one finds that the $v$ integrals can be done analytically, yielding trigonometric functions of $z_{+}$:

$$
\begin{aligned}
\int_{0}^{1} d v \hat{\pi}_{\text {scal }}^{\oplus \perp} & =\frac{1}{8 \pi}\left[-\frac{3}{2} z_{+} \operatorname{cosech}^{3} z_{+}-\frac{z_{+}^{2} \operatorname{coth}^{2} z_{+}-6 z_{+} \operatorname{coth} z_{+}+z_{+}^{2}+2}{2 z_{+} \sinh z_{+}}-\frac{2}{3}\right] \\
\int_{0}^{1} d v \hat{\pi}_{\mathrm{scal}}^{\otimes \|} & =\frac{1}{8 \pi}\left[\frac{2 \cosh z_{+}}{\sinh ^{2} z_{+}}-\frac{2}{z_{+} \sinh z_{+}}-\frac{2}{3}\right] \\
\int_{0}^{1} d v \hat{\pi}_{\mathrm{spin}}^{\oplus \perp} & =-\frac{1}{4 \pi}\left[3 \operatorname{cosech}^{2} z_{+}-\operatorname{coth} z_{+}\left(2 z_{+} \operatorname{cosech}^{2} z_{+}+\frac{1}{z_{+}}\right)+\frac{4}{3}\right] \\
\int_{0}^{1} d v \hat{\pi}_{\mathrm{spin}}^{\otimes \|} & =-\frac{1}{4 \pi}\left[2 \operatorname{cosech}^{2} z_{+}-2 \frac{\operatorname{coth} z_{+}}{z_{+}}+\frac{4}{3}\right] .
\end{aligned}
$$

Thus the remaining proper-time integrals are of the same type as the standard proper-time representations of the magnetic Euler-Heisenberg Lagrangians (see, e.g., 45]),

$$
\begin{array}{lll}
\mathcal{L}_{\text {scal }}^{\mathrm{EH}}(\hat{B})=-\frac{m^{4}}{16 \pi^{2}} \int_{0}^{\infty} \frac{d \hat{s}}{\hat{s}^{3}} \mathrm{e}^{-i \hat{s}}\left[\frac{\hat{B} \hat{s}}{\sin (\hat{B} \hat{s})}-\frac{(\hat{B} \hat{s})^{2}}{6}-1\right] & \quad \text { (Scalar QED) } \\
\mathcal{L}_{\text {spin }}^{\mathrm{EH}}(\hat{B})=\frac{m^{4}}{8 \pi^{2}} \int_{0}^{\infty} \frac{d \hat{s}}{\hat{s}^{3}} \mathrm{e}^{-i \hat{s}}\left[\frac{\hat{B} \hat{s}}{\tan (\hat{B} \hat{s})}+\frac{(\hat{B} \hat{s})^{2}}{3}-1\right] & \text { (Spinor QED) }
\end{array}
$$

In fact, they can be expressed in terms of the derivatives of these Lagrangians as follows:

$$
\begin{aligned}
\hat{\Pi}_{\text {scal,spin }}^{\oplus \perp}(\hat{\omega}=0, \hat{B}) & =-\frac{2 \pi \alpha}{m^{4}}\left(\frac{1}{\hat{B}} \frac{\partial}{\partial \hat{B}}+\frac{\partial^{2}}{\partial \hat{B}^{2}}\right) \mathcal{L}_{\text {scal,spin }}^{\mathrm{EH}}(\hat{B}), \\
\hat{\Pi}_{\text {scal,spin }}^{\otimes \|}(\hat{\omega}=0, \hat{B}) & =-\frac{4 \pi \alpha}{m^{4}} \frac{1}{\hat{B}} \frac{\partial}{\partial \hat{B}} \mathcal{L}_{\text {scal,spin }}^{\mathrm{EH}}(\hat{B}) .
\end{aligned}
$$


The existence of this connection between the photon-graviton amplitudes and the Euler-Heisenberg Lagrangians is not an accident. Eqs. (4.9) have been obtained before by Gies and Shaisultanov [46] using the linear coupling of the graviton to the Maxwell stress tensor (see eq. (A.2)), and exploiting the relation between the vacuum expectation value of the Maxwell tensor and the effective action,

$$
\left\langle T^{\mu \nu}\right\rangle=\frac{2}{\sqrt{-g}} \frac{\delta \Gamma}{\delta g_{\mu \nu}} .
$$

Applying 4.9 to the explicit representations of the Euler-Heisenberg Lagrangians in terms of the Hurwitz $\zeta$ - function [47, 48, 49, 45]

$$
\begin{aligned}
\mathcal{L}_{\text {scal }}^{\mathrm{EH}}(B)= & -\frac{(e B)^{2}}{4 \pi^{2}}\left\{\zeta_{H}^{\prime}\left(-1, \frac{1}{2}+\frac{m^{2}}{2 e B}\right)+\zeta_{H}\left(-1, \frac{1}{2}+\frac{m^{2}}{2 e B}\right)\left[1+\ln \left(\frac{m^{2}}{2 e B}\right)\right]\right. \\
& \left.+\frac{3}{4}\left(\frac{m^{2}}{2 e B}\right)^{2}\right\}, \\
\mathcal{L}_{\text {spin }}^{\mathrm{EH}}(B)= & \frac{(e B)^{2}}{2 \pi^{2}}\left\{\zeta_{H}^{\prime}\left(-1, \frac{m^{2}}{2 e B}\right)+\zeta_{H}\left(-1, \frac{m^{2}}{2 e B}\right) \ln \left(\frac{m^{2}}{2 e B}\right)-\frac{1}{12}+\frac{1}{4}\left(\frac{m^{2}}{2 e B}\right)^{2}\right\}
\end{aligned}
$$

(the prime on $\zeta_{H}$ refers to a derivative in the first variable) one obtains

$$
\begin{aligned}
& \hat{\Pi}_{\text {scal }}^{\oplus \perp}(0, \hat{B})=\frac{\alpha}{8 \pi}\left\{16 \zeta_{H}^{\prime}\left(-1, \frac{1}{2}+\frac{1}{2 \hat{B}}\right)+\frac{1}{\hat{B}^{2}} \psi\left(\frac{1}{2}+\frac{1}{2 \hat{B}}\right)-\frac{6}{\hat{B}} \ln \Gamma\left(\frac{1}{2}+\frac{1}{2 \hat{B}}\right)\right. \\
& \left.+\frac{3}{\hat{B}} \ln (2 \pi)-\frac{2}{3} \ln (2 \hat{B})-\frac{2}{\hat{B}^{2}}\right\}, \\
& \hat{\Pi}_{\text {scal }}^{\otimes \|}(0, \hat{B})=\frac{\alpha}{8 \pi}\left\{16 \zeta_{H}^{\prime}\left(-1, \frac{1}{2}+\frac{1}{2 \hat{B}}\right)-\frac{4}{\hat{B}} \ln \Gamma\left(\frac{1}{2}+\frac{1}{2 \hat{B}}\right)+\frac{2}{\hat{B}} \ln (2 \pi)\right. \\
& \left.-\frac{2}{3} \ln (2 \hat{B})-\frac{1}{\hat{B}^{2}}+\frac{1}{3}\right\} \\
& \hat{\Pi}_{\text {spin }}^{\oplus \perp}(0, \hat{B})=-\frac{\alpha}{4 \pi}\left\{16 \zeta_{H}^{\prime}\left(-1, \frac{1}{2 \hat{B}}\right)-\frac{6}{\hat{B}} \ln \Gamma\left(\frac{1}{2 \hat{B}}\right)+\frac{1}{\hat{B}^{2}} \psi\left(\frac{1}{2 \hat{B}}\right)\right. \\
& \left.+\frac{1}{\hat{B}} \ln \left(\frac{4 \pi^{3}}{\hat{B}}\right)+\frac{4}{3} \ln (2 \hat{B})-\frac{2}{\hat{B}^{2}}+\frac{1}{\hat{B}}\right\}, \\
& \hat{\Pi}_{\text {spin }}^{\otimes \|}(0, \hat{B})=-\frac{\alpha}{4 \pi}\left\{16 \zeta_{H}^{\prime}\left(-1, \frac{1}{2 \hat{B}}\right)-\frac{4}{\hat{B}} \ln \Gamma\left(\frac{1}{2 \hat{B}}\right)-\frac{2}{\hat{B}} \ln (\hat{B} / \pi)\right. \\
& \left.+\frac{4}{3} \ln (2 \hat{B})-\frac{1}{\hat{B}^{2}}-\frac{2}{3}\right\} \text {. }
\end{aligned}
$$


Here $\psi(x)=\Gamma^{\prime}(x) / \Gamma(x)$ is the digamma function.

\subsection{Weak magnetic field and arbitrary photon energy}

We proceed to the case of arbitrary photon energy but weak magnetic field, i.e., $B \ll B_{\text {cr. }}$. Our treatment of this case parallels the one introduced by Tsai and Erber for the photon-photon case [29] (see also [32]).

Assuming $\hat{B} \ll 1$, we can expand the trigonometric functions appearing in the integrands (4.3), 4.4), in the common exponential factor (which is the same as in the photon-photon case [38]) as well as in the prefactor functions, keeping only the terms of lowest order in $z_{+}$. However, since we do not wish to make any assumption on the ratio $\frac{\hat{\omega}^{2}}{\hat{B}}$, this truncation has to be done separately for the terms with and without a factor of $\frac{\hat{\omega}^{2}}{\hat{B}}$. For example, the prefactor of $\hat{\pi}_{\text {scal }}^{\oplus \perp}$ involves (see eq. 4.3 )

$$
\begin{aligned}
& \left(S_{B 12}^{+}\right)^{2}-\bar{A}_{B 12}^{+}\left(\bar{A}_{B 12}^{+}+\frac{1}{z_{+}}\right)+\bar{A}_{B 12}^{+}\left(\left(S_{B 12}^{+}\right)^{2}-\left(\bar{A}_{B 12}^{+}\right)^{2}-v S_{B 12}^{+}\right) \frac{\hat{\omega}^{2}}{2 \hat{B}} \\
& =\frac{1}{2}+\frac{v^{2}}{2}+O\left(z_{+}^{2}\right)+\frac{\hat{\omega}^{2}}{\hat{B}}\left[\frac{1}{48}\left(v^{2}-1\right)^{2}\left(3-v^{2}\right) z_{+}^{3}+O\left(z_{+}^{5}\right)\right] .
\end{aligned}
$$

Although the first term in the square bracket is $O\left(z_{+}^{3}\right)$, at this stage it cannot be neglected over the leading $\frac{1}{2}+\frac{v^{2}}{2}$ term. Similarly, the expansion of the universal exponent in eqs. 4.3, (4.4) yields

$$
z_{+}\left(\frac{\bar{A}_{B 12}^{+}}{z_{+}}+\frac{1}{2}\left(1-v^{2}\right)\right) \frac{\hat{\omega}^{2}}{2 \hat{B}}=\frac{1}{48}\left(1-v^{2}\right)^{2} \frac{\hat{\omega}^{2}}{\hat{B}} z_{+}^{3}+O\left(z_{+}^{5}\right) .
$$

Here the leading order term, although of order $O\left(z_{+}^{3}\right)$, cannot be neglected with respect to the exponent of the global factor $\mathrm{e}^{-i \hat{s}}$.

After performing these truncations, the amplitude ratios turn out to depend on $\hat{B}$ and $\hat{\omega}$ only in the combination $\hat{B} \hat{\omega}$. This motivates the introduction of a new parameter $\lambda$,

$$
\lambda \equiv \frac{3}{2} \hat{B} \hat{\omega} .
$$

Moreover, for the following it will be useful to interchange the orders of integrations in (3.5), and perform a $v$-dependent change of variables of the global proper-time variable from $\hat{s}$ to $x$, 


$$
\hat{s}=\rho x
$$

where

$$
\rho=\left(\frac{6}{\lambda\left(1-v^{2}\right)}\right)^{\frac{2}{3}}
$$

After this truncation and change of variables, the amplitude ratios take the following form:

$$
\begin{aligned}
\hat{\Pi}_{\text {scal }}^{\oplus \perp}(\lambda)= & \frac{\alpha}{8 \pi} \operatorname{Re} \int_{0}^{1} d v \int_{0}^{\infty} \frac{d x}{x} \\
& \times\left[\frac{1}{2}\left(v^{2}+1\right)\left(\mathrm{e}^{-i \Xi}-\mathrm{e}^{-i \rho x}\right)-\frac{i}{3}\left(3-v^{2}\right) x^{3} \mathrm{e}^{-i \Xi}\right], \\
\hat{\Pi}_{\mathrm{scal}}^{\otimes \|}(\lambda)= & \frac{\alpha}{8 \pi} \operatorname{Re} \int_{0}^{1} d v \int_{0}^{\infty} \frac{d x}{x} \\
& \times\left[\frac{1}{2}\left(v^{2}+1\right)\left(\mathrm{e}^{-i \Xi}-\mathrm{e}^{-i \rho x}\right)-i \frac{2}{3} v^{2} x^{3} \mathrm{e}^{-i \Xi}\right], \\
\hat{\Pi}_{\mathrm{spin}}^{\oplus \perp}(\lambda)= & -\frac{\alpha}{4 \pi} \operatorname{Re} \int_{0}^{1} d v \int_{0}^{\infty} \frac{d x}{x} \\
& \times\left[\frac{1}{2}\left(v^{2}-3\right)\left(\mathrm{e}^{-i \Xi}-\mathrm{e}^{-i \rho x}\right)+\frac{i}{3}\left(3+v^{2}\right) x^{3} \mathrm{e}^{-i \Xi}\right], \\
\hat{\Pi}_{\mathrm{spin}}^{\otimes \|}(\lambda)= & -\frac{\alpha}{4 \pi} \operatorname{Re} \int_{0}^{1} d v \int_{0}^{\infty} \frac{d x}{x} \\
& \times\left[\frac{1}{2}\left(v^{2}-3\right)\left(\mathrm{e}^{-i \Xi}-\mathrm{e}^{-i \rho x}\right)+i \frac{2}{3}\left(3-v^{2}\right) x^{3} \mathrm{e}^{-i \Xi}\right] .
\end{aligned}
$$

The common exponential factor $\mathrm{e}^{-i \Xi}$ is now of the Airy form,

$$
\Xi=\rho x+\frac{1}{3} x^{3}
$$

Note that we have also rewritten the counterterms in a way which will be convenient in the following. 
Now, the two different $x$ - integrals appearing here can both be expressed in terms of the modified Airy function Gi( $\rho)$, defined by

$$
G i(\rho) \equiv \frac{1}{\pi} \int_{0}^{\infty} d x \sin \left(\rho x+\frac{1}{3} x^{3}\right)
$$

Namely, one has

$$
\begin{aligned}
f_{1}(\rho) & \equiv \int_{0}^{\infty} \frac{d x}{x}\left[\cos \left(\rho x+\frac{1}{3} x^{3}\right)-\cos (\rho x)\right] \\
& =\int_{0}^{\infty} \frac{d x}{x}\left[\cos \left(\frac{1}{3} x^{3}\right)-x \int_{0}^{\rho} d \rho^{\prime} \sin \left(\rho^{\prime} x+\frac{1}{3} x^{3}\right)-\cos (\rho x)\right] \\
& =-\pi \int_{0}^{\rho} d \rho^{\prime} G i\left(\rho^{\prime}\right)+\ln \rho+\frac{2}{3} \gamma+\frac{1}{3} \ln 3,
\end{aligned}
$$

and

$$
f_{2}(\rho) \equiv \int_{0}^{\infty} d x x^{2} \sin \left(\rho x+\frac{1}{3} x^{3}\right)=-\pi G i^{\prime \prime}(\rho)=1-\pi \rho G i(\rho)
$$

This brings us to our final result,

$$
\begin{aligned}
\hat{\Pi}_{\text {scal }}^{\oplus \perp}(\lambda) & =\frac{\alpha}{8 \pi} \int_{0}^{1} d v\left[\frac{1}{2}\left(v^{2}+1\right) f_{1}(\rho)-\frac{1}{3}\left(3-v^{2}\right) f_{2}(\rho)\right], \\
\hat{\Pi}_{\text {scal }}^{\otimes \|}(\lambda) & =\frac{\alpha}{8 \pi} \int_{0}^{1} d v\left[\frac{1}{2}\left(v^{2}+1\right) f_{1}(\rho)-\frac{2}{3} v^{2} f_{2}(\rho)\right], \\
\hat{\Pi}_{\text {spin }}^{\oplus \perp}(\lambda) & =-\frac{\alpha}{4 \pi} \int_{0}^{1} d v\left[\frac{1}{2}\left(v^{2}-3\right) f_{1}(\rho)+\frac{1}{3}\left(3+v^{2}\right) f_{2}(\rho)\right], \\
\hat{\Pi}_{\text {spin }}^{\otimes \|}(\lambda) & =-\frac{\alpha}{4 \pi} \int_{0}^{1} d v\left[\frac{1}{2}\left(v^{2}-3\right) f_{1}(\rho)+\frac{2}{3}\left(3-v^{2}\right) f_{2}(\rho)\right] .
\end{aligned}
$$

Using 4.21, 4.22 these integrals can be done numerically without difficulties for any value of $\lambda$. 
As in the photon-photon case [29], exact results can be obtained in the limits of small and large $\lambda$. Using the known asymptotic properties of the function $G i(x)$ [50, 51] it is easy to show that, for small $\rho$,

$$
\begin{aligned}
& f_{1}(\rho) \sim \ln (\rho)+\frac{2}{3} \gamma+\frac{1}{3} \ln 3+O(\rho) \\
& f_{2}(\rho)=1+O(\rho)
\end{aligned}
$$

while for $\rho \rightarrow \infty$

$$
\begin{aligned}
& f_{1}(\rho) \sim \frac{2}{3} \frac{1}{\rho^{3}}+O\left(\frac{1}{\rho^{6}}\right), \\
& f_{2}(\rho) \sim-\frac{2}{\rho^{3}}+O\left(\frac{1}{\rho^{6}}\right) .
\end{aligned}
$$

Using the leading terms of the expansions $(4.25)$ in 4.23 yields the following results for the leading order terms of the amplitude ratios in the small $\lambda$ limit:

$$
\begin{aligned}
\hat{\Pi}_{\mathrm{scal}}^{\oplus \perp}(\lambda) & =\frac{\alpha}{8 \pi} \frac{32}{945} \lambda^{2}+O\left(\lambda^{4}\right), \\
\hat{\Pi}_{\mathrm{scal}}^{\otimes \|}(\lambda) & =\frac{\alpha}{8 \pi} \frac{8}{945} \lambda^{2}+O\left(\lambda^{4}\right), \\
\hat{\Pi}_{\mathrm{spin}}^{\oplus \perp}(\lambda) & =\frac{\alpha}{4 \pi} \frac{128}{2835} \lambda^{2}+O\left(\lambda^{4}\right), \\
\hat{\Pi}_{\mathrm{spin}}^{\otimes \|}(\lambda) & =\frac{\alpha}{4 \pi} \frac{40}{567} \lambda^{2}+O\left(\lambda^{4}\right) .
\end{aligned}
$$

Using the leading terms of the expansions 4.24 gives the asymptotic behaviour for large $\lambda$ :

$$
\begin{aligned}
\hat{\Pi}_{\text {scal }}^{\oplus \perp}(\lambda) & =\frac{\alpha}{8 \pi}\left[-\frac{4}{9} \ln (\lambda)+\frac{4}{9} \gamma+\frac{2}{3} \ln 3-\frac{4}{9} \ln 2+\frac{2}{27}+O\left(\lambda^{-\frac{2}{3}}\right)\right], \\
\hat{\Pi}_{\text {scal }}^{\otimes \|}(\lambda) & =\frac{\alpha}{8 \pi}\left[-\frac{4}{9} \ln (\lambda)+\frac{4}{9} \gamma+\frac{2}{3} \ln 3-\frac{4}{9} \ln 2+\frac{20}{27}+O\left(\lambda^{-\frac{2}{3}}\right)\right], \\
\hat{\Pi}_{\text {spin }}^{\oplus \perp}(\lambda) & =\frac{\alpha}{4 \pi}\left[-\frac{8}{9} \ln (\lambda)+\frac{8}{9} \gamma+\frac{4}{3} \ln 3-\frac{8}{9} \ln 2+\frac{16}{27}+O\left(\lambda^{-\frac{2}{3}}\right)\right], \\
\hat{\Pi}_{\text {spin }}^{\otimes \|}(\lambda) & =\frac{\alpha}{4 \pi}\left[-\frac{8}{9} \ln (\lambda)+\frac{8}{9} \gamma+\frac{4}{3} \ln 3-\frac{8}{9} \ln 2-\frac{2}{27}+O\left(\lambda^{-\frac{2}{3}}\right)\right] .
\end{aligned}
$$


Note that the leading logarithmic terms in 4.27 are independent of the polarization choice. As in the case of the leading asymptotic growth for large field strength, eq. (4.6), their coefficients are directly related to the UV counterterms. This is another fact which is familiar from the photon-photon case. Fig. 3 shows the result of a numerical evaluation, using MATHEMATICA, of the parameter integral 4.23 for the amplitude $\hat{\Pi}_{\text {spin }}^{\oplus \perp}(\lambda)$. The small and large $\lambda$ approximations (4.26), 4.27) are also shown.

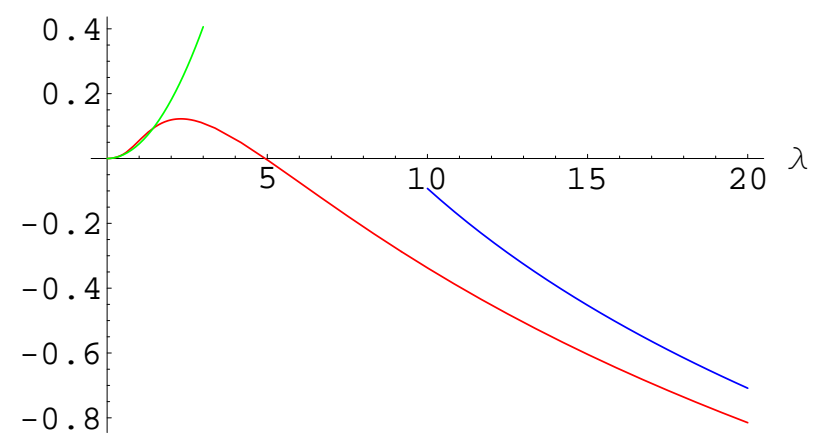

Figure 3: Numerical plot of the amplitude $\hat{\Pi}_{\text {spin }}^{\oplus \perp}(\lambda)$ for $\lambda$ between 0 and 20 . The small and large $\lambda$ approximations are also shown. A global factor of $\frac{\alpha}{4 \pi}$ has been omitted.

\section{The electric field case}

The photon-graviton conversion process in a constant field seems to have been studied hitherto exclusively for the magnetic field case. The reason is, of course, the absence of evidence for the existence of very strong macroscopic electric fields anywhere in nature. Still, in the near future it may be possible to reach field strengths of the order of $E_{\mathrm{cr}}$ in the laboratory using optical or X-ray lasers [52, 53, 54]. Thus it seems worthwhile to shortly discuss also the electric case. Moreover, some of our results for the magnetic case carry over to the electric case simply by analytic continuation. Namely, electric-magnetic duality for this amplitude takes the following form (see eqs.2.14 and (2.19), 


$$
\begin{aligned}
& \hat{\Pi}_{\text {scal,spin }}^{\oplus \|}(\hat{\omega}, 0, \hat{E})=\left.\hat{\Pi}_{\text {scal,spin }}^{\oplus \perp}(\hat{\omega}, \hat{B}, 0)\right|_{B \rightarrow i E, \omega \rightarrow-i \omega} \\
& \hat{\Pi}_{\text {scal,spin }}^{\otimes \perp}(\hat{\omega}, 0, \hat{E})=\left.\hat{\Pi}_{\text {scal,spin }}^{\otimes \|}(\hat{\omega}, \hat{B}, 0)\right|_{B \rightarrow i E, \omega \rightarrow-i \omega}
\end{aligned}
$$

Despite of this formal duality, there is a structural difference between the electric and magnetic amplitudes. While the magnetic amplitude ratios are real for photon energies below the pair creation threshold, the integrands of the corresponding electric ones have poles in the Euclidean proper-time variable $T=i s$, indicating the existence of an imaginary part for all photon energies. This again agrees with the photon-photon case [41, and physically corresponds to the fact that both the photon/graviton and the electric field are capable of pair production, while in the magnetic case the field alone cannot induce an absorptive part. Although we are interested here only in the real parts, technically this implies that a calculation of the full electric amplitudes through numerical computation of the parameter integrals (3.5) is even more difficult than for the magnetic amplitudes. In particular, the rotation to Euclidean proper-time is much less useful here, even for $\omega$ below threshold, since the rotated integrand has poles, both in the prefactor functions and in the exponent. We will therefore restrict our discussion to two limiting cases where we can directly draw on our results for the magnetic case, the zero photon energy case and the weak field case.

\subsection{Zero photon energy and arbitrary electric field strength}

For $\omega=0$, in eqs. 4.12 we have the magnetic amplitudes in an explicit form involving only the functions $\Gamma, \psi$, and $\zeta_{H}$. Implementing the analytic continuation $B \rightarrow i E$ is therefore straightforward. In particular, for large $E$ the amplitude ratio shows the same logarithmic growth as we had found in the magnetic case (eq. 4.6 ),

$$
\begin{aligned}
& \hat{\Pi}_{\text {scal }}^{A a}(\hat{\omega}=0, \hat{E}) \quad \stackrel{\hat{E} \rightarrow \infty}{\sim}-\frac{\alpha}{12 \pi} \ln (\hat{E}), \\
& \hat{\Pi}_{\text {spin }}^{A a}(\hat{\omega}=0, \hat{E}) \quad \stackrel{\hat{E} \rightarrow \infty}{\sim}-\frac{\alpha}{3 \pi} \ln (\hat{E}) .
\end{aligned}
$$




\subsection{Weak electric field and arbitrary photon energy}

Things become even simpler in the weak field limit. The approximation used in section 4.3 , based on a weak field expansion and truncation to the leading order terms, removes the additional poles contained in the electric integrand, leading to a complete symmetry between the electric and magnetic cases. Since in this approximation the amplitude ratios depend only on the single variable $\lambda=\frac{3}{2} \hat{B} \hat{\omega}$, eqs. 5.1 turn into

$$
\begin{aligned}
\hat{\Pi}_{\mathrm{scal}, \text { spin }}^{\oplus \|}\left(\lambda=\frac{3}{2} \hat{E} \hat{\omega}\right) & =\hat{\Pi}_{\mathrm{scal}, \mathrm{spin}}^{\oplus \perp}\left(\lambda=\frac{3}{2} \hat{B} \hat{\omega}\right), \\
\hat{\Pi}_{\mathrm{scal}, \text { spin }}^{\otimes \perp}\left(\lambda=\frac{3}{2} \hat{E} \hat{\omega}\right) & =\hat{\Pi}_{\mathrm{scal}, \mathrm{spin}}^{\otimes \|}\left(\lambda=\frac{3}{2} \hat{B} \hat{\omega}\right) .
\end{aligned}
$$

Therefore all the results of section 4.3 carry over to the electric case mutatis mutandis.

\section{Conclusions}

We have analyzed the parameter integral representations obtained in part 1 for the one-loop photon-graviton amplitudes in a constant field at about the same level of detail as was previously achieved for the analogous QED photon-photon case [27, 29, 31, 32, 33, 41]. In the purely magnetic case, we have shown that our representation is amenable to a direct numerical evaluation for photon/graviton energies below threshold, at arbitrary field strength. For weak magnetic fields, a one-parameter integral representation involving Airy functions has been obtained, and shown to be suitable to numerical evaluation. Closed-form results have been found for the zero energy limit. We have also transformed our results for the weak field and zero energy magnetic cases to corresponding results for the electric field case.

The qualitative properties of the photon-graviton amplitudes turn out to be closely analogous to the ones of the corresponding photon-photon amplitudes. In particular, in the magnetic case they have the same same pair creation thresholds, and a similar asymptotic growth for strong fields or large photon/graviton energies. This is, of course, not surprising, particularly considering that the photon-photon and photon-graviton amplitudes are connected by the gravitational Ward identity ((A.13) of part 1). Apart from the pair creation thresholds (4.5), none of our results show any substantial differences between the scalar and spinor loop cases. 
From a quantitative point of view, our analysis of the one-loop photongraviton amplitude can be summarized as follows. For small photon energies and large magnetic or electric field strengths, this ratio grows logarithmically in the field strength (see (4.6) resp. (5.2)). For weak fields and large photon energies it grows logarithmically with the photon energy (see 4.27), (5.3)). In these limits it is clearly not possible to compensate the small prefactor $\alpha / 4 \pi$ for physically relevant values of the parameters. While we have not been able to perform a quantitative analysis in the whole twoparameter space of field strengths and energies, a compound effect of large field strengths and large energies appears to be exluded by the fact that the integrand of 3.5 contains $\omega$ only in the combination $\hat{\omega}^{2} / \hat{B}$ resp. $\hat{\omega}^{2} / \hat{E}$. This is also borne out by the numerical results of section 4.1. Overall, we conclude that the magnitude of the one-loop contribution to this amplitude will not amount to more than a few percent of the tree level one for physically realistic values of the parameters.

It should be mentioned, though, that there is also a qualitative difference to the tree-level amplitude. As has been stressed in [10, the tree level photon-graviton conversion does, contrary to the photon-axion case, not lead to a dichroism effect for photon beams. This is because, according to (2.14), both photon polarization components have equal conversion rates. This symmetry does not extend to the one-loop level (except for the strong field limit).

As we have seen, in the worldline formalism the calculation of the photongraviton polarization tensor in a constant field is only moderately more difficult than the one of the photon-photon polarization tensor [38. We expect that even the graviton-graviton case will be quite feasible. In a future sequel, we intend to analyze this case at the same level of the photongraviton one, which then would make it possible to study the complete set of one-loop photon-graviton dispersion relations (1.4).

Acknowledgements: We thank J. Ehlers, H. Gies, G. Raffelt, and S. Theisen for helpful discussions. F.B., C.S., and V. V. thank the AlbertEinstein Institute, Potsdam, for hospitality during part of this work. C. S. and V. V. thank CONACYT for support through grant PROYECTO 38293-E. 


\section{A Quadratic expansion of the Einstein-Maxwell theory}

The Einstein-Maxwell theory is described by

$$
S[g, A]=\int d^{D} x \sqrt{g}\left(\frac{1}{\kappa^{2}} R-\frac{1}{4} F_{\mu \nu} F^{\mu \nu}\right)
$$

where the metric $g_{\mu \nu}$ has signature $(-,+,+, \ldots,+), g=\left|\operatorname{det} g_{\mu \nu}\right|$, and $\kappa^{2}=$ $16 \pi G_{N}$. The spacetime dimension of interest is $D=4$, but in this section we may as well keep it arbitrary. We expand $g_{\mu \nu}=\eta_{\mu \nu}+\kappa h_{\mu \nu}$ and $A_{\mu}=\bar{A}_{\mu}+a_{\mu}$. Then using the short-hand notation $h_{\mu} \equiv \partial^{\alpha} h_{\alpha \mu}$ and $h \equiv \eta^{\mu \nu} h_{\mu \nu}$ one obtains the following quadratic approximation in the fluctuations $\left(h_{\mu \nu}, a_{\mu}\right)$ around the background $\left(\eta_{\mu \nu}, \bar{A}_{\mu}\right)$

$$
\begin{aligned}
S_{(2)} & =\int d^{D} x\left\{\frac{1}{4}\left(h^{\mu \nu} \square h_{\mu \nu}-h \square h+2 h \partial^{\mu} h_{\mu}+2 h^{\mu} h_{\mu}\right)+\frac{1}{2}\left(a^{\mu} \square a_{\mu}+\left(\partial_{\mu} a^{\mu}\right)^{2}\right)\right. \\
& +\frac{\kappa}{2} h_{\mu \nu}\left(\bar{F}^{\mu \alpha} \bar{F}^{\nu}{ }_{\alpha}-\frac{1}{4} \eta^{\mu \nu} \bar{F}^{2}\right) \\
& +\kappa h_{\mu \nu}\left(\bar{F}^{\mu \alpha} f^{\nu}{ }_{\alpha}-\frac{1}{4} \eta^{\mu \nu} \bar{F}^{\alpha \beta} f_{\alpha \beta}\right) \\
& \left.-\frac{\kappa^{2}}{4}\left[\left(\frac{1}{8} h^{2}-\frac{1}{4} h_{\mu \nu}^{2}\right) \bar{F}^{2}+h^{\mu \nu} h^{\alpha \beta} \bar{F}_{\mu \alpha} \bar{F}_{\nu \beta}+\left(2 h^{\mu \alpha} h^{\nu}{ }_{\alpha}-h h^{\mu \nu}\right) \bar{F}_{\mu \beta} \bar{F}_{\nu}{ }^{\beta}\right]\right\}
\end{aligned}
$$

In the second line of this expression we recognize the linear coupling $\frac{\kappa}{2} h_{\mu \nu} \bar{T}^{\mu \nu}$ of the graviton $h_{\mu \nu}$ with the stress tensor of the background electromagnetic field $\bar{T}^{\mu \nu}=\bar{F}^{\mu \alpha} \bar{F}^{\nu}{ }_{\alpha}-\frac{1}{4} \eta^{\mu \nu} \bar{F}^{2}$. This tadpole vertex indicates that the nontrivial background stress tensor tends to curve the space. The third line in A.2 gives instead the tree level graviton-photon mixing in the electromagnetic background. Using plane waves

$$
h_{\mu \nu}(x)=\epsilon_{\mu \nu} e^{i k x}, \quad a_{\alpha}(x)=\epsilon_{\alpha} e^{i k_{2} x}
$$

we get for this mixing term the vertex

$$
\Delta S_{(2)}=(2 \pi)^{D} \delta^{D}\left(k+k_{2}\right) \epsilon_{\mu \nu} \epsilon_{\alpha}(-i \kappa)\left[F^{\mu \alpha} k^{\nu}-(F \cdot k)^{\mu} \eta^{\nu \alpha}+\frac{1}{2} \eta^{\mu \nu}(F \cdot k)^{\alpha}\right]
$$

which appears in the path integrals as $e^{i \Delta S_{(2)}}$ (see eq. (1.2)). 
The two-point functions (which we denote by $\Pi$ ), in either coordinate or momentum space, are contained in $S_{(2)}$ (or in the quadratic part of the full effective action $\left.\Gamma_{(2)}=S_{(2)}+\Gamma_{(2)}^{(1-\text { loop })}+\cdots\right)$ as follows

$$
\begin{aligned}
S_{(2)}= & \int d^{D} x\left\{\frac{1}{2} h_{\mu \nu}(x) \Pi^{\mu \nu, \lambda \rho}(\partial) h_{\lambda \rho}(x)+\frac{1}{2} a_{\alpha}(x) \Pi^{\alpha, \beta}(\partial) a_{\beta}(x)\right. \\
& \left.+h_{\mu \nu}(x) \Pi^{\mu \nu, \alpha}(\partial) a_{\alpha}(x)+\frac{\kappa}{2} h_{\mu \nu}(x) \bar{T}^{\mu \nu}(x)\right\} \\
= & \int \frac{d^{D} k}{(2 \pi)^{D}}\left\{\frac{1}{2} h_{\mu \nu}(k) \Pi^{\mu \nu, \lambda \rho}(k) h_{\lambda \rho}(-k)+\frac{1}{2} a_{\alpha}(k) \Pi^{\alpha, \beta}(k) a_{\beta}(-k)\right. \\
& \left.+h_{\mu \nu}(k) \Pi^{\mu \nu, \alpha}(k) a_{\alpha}(-k)+\frac{\kappa}{2} h_{\mu \nu}(k) \bar{T}^{\mu \nu}(-k)\right\}
\end{aligned}
$$

where the Fourier transform of a field is given by $h_{\mu \nu}(x)=\int \frac{d^{D} k}{(2 \pi)^{D}} e^{i k x} h_{\mu \nu}(k)$, its inverse by $h_{\mu \nu}(k)=\int d^{D} x e^{-i k x} h_{\mu \nu}(x)$ and $\Pi(k)=\Pi(\partial \rightarrow-i k)$.

The equations of motion in term of these two-point functions then read ${ }^{3}$

$$
\begin{aligned}
\delta a_{\alpha}(k): & \Pi^{\alpha, \beta}(k) a_{\beta}(-k)+\Pi^{\mu \nu, \alpha}(-k) h_{\mu \nu}(-k)=0 \\
\delta h_{\mu \nu}(k): & \Pi^{\mu \nu, \lambda \rho}(k) h_{\lambda \rho}(-k)+\Pi^{\mu \nu, \alpha}(k) a_{\alpha}(-k)=-\frac{\kappa}{2} \bar{T}^{\mu \nu}(-k)
\end{aligned}
$$

and, in particular, one obtains from A.2

$$
\begin{aligned}
& \Pi_{\text {tree }}^{\alpha, \beta}(k)=k^{\alpha} k^{\beta}-k^{2} \eta^{\alpha \beta} \\
& \Pi_{\text {tree }}^{\mu \nu, \alpha}(k)=-\frac{i \kappa}{2} C^{\mu \nu, \alpha}(k)
\end{aligned}
$$

with $C^{\mu \nu, \alpha}(k)$ as in 1.2 . Also, one gets

$$
\begin{aligned}
\Pi_{\text {tree }}^{\mu \nu, \lambda \rho}(k)= & \frac{k^{2}}{2}\left(\eta^{\mu \nu} \eta^{\lambda \rho}-\frac{1}{2}\left(\eta^{\mu \lambda} \eta^{\nu \rho}+\eta^{\mu \rho} \eta^{\nu \lambda}\right)\right)-\frac{1}{2}\left(\eta^{\mu \nu} k^{\lambda} k^{\rho}+k^{\mu} k^{\nu} \eta^{\lambda \rho}\right) \\
& +\frac{1}{4}\left(\eta^{\mu \lambda} k^{\nu} k^{\rho}+\eta^{\nu \lambda} k^{\mu} k^{\rho}+\eta^{\mu \rho} k^{\nu} k^{\lambda}+\eta^{\nu \rho} k^{\mu} k^{\lambda}\right)+\bar{F} \text { terms. }
\end{aligned}
$$

Finally, note that a constant $\bar{T}^{\mu \nu}$ gives $\bar{T}^{\mu \nu}(k)=(2 \pi)^{2} \delta^{D}(k) \bar{T}^{\mu \nu}$.

\footnotetext{
${ }^{3}$ A minus sign in the euclidean formulas (A.11) of paper I is correctly taken into account by the Wick rotation, as one can easily check by looking at the euclidean tree level Maxwell action $S=\int d^{D} x \frac{1}{4} F_{\mu \nu} F^{\mu \nu}$, which indeed is positive definite in euclidean space (as it should).
} 


\section{References}

[1] M.E. Gertsenshtein, Sov. Phys. JETP 14 (1962) 84.

[2] Ya. B. Zel'dovich and I.D. Novikov, The structure and evolution of the universe, Rel. Astrophys. Vol. 2 (1983), Chicago University Press.

[3] S.M. Bilenky and B. Pontecorvo, Phys. Rep. 41 (1978) 225.

[4] H. Primakoff, Phys. Rev. 81 (1951) 899.

[5] D.A. Dicus, E.W. Kolb, V.L. Teplitz, and R.V. Wagoner, Phys. Rev. D 18 (1978) 1829.

[6] G.G. Raffelt and L. Stodolsky, Phys. Rev. D 37 (1988) 1237.

[7] H.N. Long, D.V. Soa, and T.A. Tran, Mod. Phys. Lett. A9 (1994) 3619, astro-ph/9410003.

[8] J.C.R. Magueijo, Phys. Rev. D 49 (1994) 671.

[9] P. Chen, Phys. Rev. Lett. 74 (1995) 634; erratum ibid. 74 (1995) 3091.

[10] A.N. Cillis and D. Harari, Phys. Rev. D 54 (1996) 4757, astro$\mathrm{ph} / 9609200$.

[11] G.G. Raffelt, Stars as Laboratories for Fundamental Physics, Chicago University Press 1996.

[12] P. Sikivie, Phys. Rev. Lett. 51 (1983) 1415; erratum ibid. 52 (1984) 695.

[13] D. E. Morris, Phys. Rev. D 34 (1986) 843.

[14] C. Csáki, N. Kaloper and J. Terning, Phys. Rev. Lett. 88 (2002) 161302, hep-ph/0111311; Phys. Lett. B 535 (2002) 33, hep-ph/0112212.

[15] Y. Grossman, S. Roy and J. Zupan, Phys. Lett. B 543 (2002) 23, hep$\mathrm{ph} / 0204216$.

[16] E. Mortsell, L. Bergstrom and A. Goobar, Phys. Rev. D 66 (2002) 047702, astro-ph/0202153.

[17] C. Csáki, N. Kaloper, M. Peloso and J. Terning, JCAP 0305 (2003) 005, hep-ph/0302030. 
[18] B.A. Bassett and M. Kunz, Astrophys. J. 607 (2004) 661, astro$\mathrm{ph} / 0311495$.

[19] N. Arkani-Hamed, S. Dimopoulos, and G. Dvali, Phys. Lett. B429 (1998) 263, hep-ph/9803315; Phys. Rev. D59 (1999) 086004, hep$\mathrm{ph} / 9807344$.

[20] C. Deffayet and J.-P. Uzan, Phys. Rev. D62 (2000) 063507, hep$\mathrm{ph} / 0002129$.

[21] F. Bastianelli and C. Schubert, JHEP 0502 (2005) 069, gr-qc/0412095.

[22] J.S. Toll, Ph D thesis, Princeton Univ., 1952 (unpublished).

[23] A. Minguzzi, Nuovo Cim. 6 (1956) 476.

[24] R. Baier and P. Breitenlohner, Acta Phys. Austr. 25 (1967) 212; Nuovo Cim. 47 (1967) 261.

[25] Z. Bialynicka-Birula and I. Bialynicka-Birula, Phys. Rev. D 2 (1970) 2341.

[26] S.L. Adler, Ann. Phys. (N.Y.) 67 (1971) 599.

[27] I.A. Batalin and A.E. Shabad, Zh. Eksp. Teor. Fiz. 60 (1971) 894 [JETP 33 (1971) 483].

[28] V.I. Ritus, Ann. Phys. (N.Y.) 69 (1972) 555.

[29] W.-Y. Tsai and T. Erber, Phys. Rev. D 10 (1974) 492; Phys. Rev. D 12 (1975) 1132.

[30] V.N. Baier, V.M. Katkov, and V.M. Strakhovenko, Zh. Eksp. Teor. Fiz. 68 (1975) 405 [JETP 41 (1975) 198].

[31] D.B. Melrose and R.J. Stoneham, Nuov. Cim. 32 (1976) 435.

[32] W. Dittrich and H. Gies, Probing the Quantum Vacuum, Springer 2000.

[33] K. Kohri and S. Yamada, Phys. Rev. D 65:043006 (2002), astro$\mathrm{ph} / 0102225$.

[34] I.T. Drummond and S.J. Hathrell, Phys. Rev. D 22 (1980) 343.

[35] J.I. Latorre, P. Pascual, and R. Tarrach, Nucl. Phys. B 437 (1995) 60, hep-th/9408016. 
[36] G.M. Shore, Nucl. Phys. B 460379 (1996), gr-qc/9504041; Nucl. Phys. B 778 (2007) 219, hep-th/0701185.

[37] T.J. Hollowood and G.M. Shore, arXiv:0707.2302 [hep-th]; arXiv:0707.2303 [hep-th].

[38] C. Schubert, Nucl. Phys. B 585 (2000) 407, hep-ph/0001288.

[39] C. Schubert, Phys. Rept. 355 (2001) 73, hep-th/0101036.

[40] R.C. Duncan and C. Thompson, Astrophys. J. 392:L9 (1992).

[41] G.K. Artimovich, Zh. Eksp. Teor. Fiz. 97 (1990) 1393 [JETP 70 (1990) 787].

[42] W.-F. Kao, G.-L. Lin, and J.-J. Tseng, Phys. Lett. B 522 (2001) 257, hep-ph/0109002; Erratum ibid. B 541 (2002) 411.

[43] V. I. Ritus, Zh. Eksp. Teor. Fiz. 73 (1977) 807 [JETP 46 (1977) 423].

[44] G.V. Dunne, H. Gies, and C. Schubert, JHEP 0211 (2002) 032, hepth/0210240.

[45] G. V. Dunne, Heisenberg-Euler effective Lagrangians: Basics and extensions, in Ian Kogan Memorial Collection, From Fields to Strings: Circumnavigating Theoretical Physics, M. Shifman et al (Eds) (World Scientific, Singapore, 2004), Volume 1, pp. 445-522, hep-th/0406216.

[46] H. Gies and R. Shaisultanov, unpublished work (private communication by H. Gies).

[47] W. Dittrich, J. Phys. A 9 (1976) 1171.

[48] W. Dittrich and M. Reuter, Effective Lagrangians in quantum electrodynamics, Springer Lecture Notes in Physics Vol. 220, Springer 1985.

[49] S. K. Blau, M. Visser, and A. Wipf, Int. J. Mod. Phys. A 6 (1991) 5409.

[50] F. W. J. Olver, Asymptotics and Special Functions, Acad. Press, London 1974.

[51] A.I. Nikishov and V.I. Ritus, math-ph/0501062.

[52] J. Hein et al., Appl. Phys. B 79 (2004) 419. 
[53] Linac Coherent Light Source, http://www-ssrl.slac.stanford.edu/lcls/

[54] European X-Ray Laser Project XFEL, http://xfel.desy.de/ 